\title{
ON A CONJECTURE OF BELTRAMETTI-SOMMESE FOR POLARIZED 4-FOLDS
}

\author{
YOSHIAKI FUKUMA
}

\begin{abstract}
Let $(X, L)$ be a polarized manifold of dimension 4. In this paper, we prove that $h^{0}\left(K_{X}+3 L\right)>0$ if $K_{X}+3 L$ is nef, which is a conjecture of Beltrametti-Sommese for polarized 4-folds.
\end{abstract}

\section{Introduction}

Let $X$ be a projective variety of dimension $n$ defined over the field of complex numbers, and let $L$ be an ample line bundle on $X$. Then $(X, L)$ is called a polarized variety. If $X$ is smooth, then we say that $(X, L)$ is a polarized manifold.

The adjoint bundle $K_{X}+(n-1) L$ of $(X, L)$ plays an important role for investigating $(X, L)$ (for example, see [1, Chapter 7, 9, and 11]), where $K_{X}$ is the canonical line bundle. conjecture.

In [1, Conjecture 7.2.7], Beltrametti and Sommese gave the following

CONJeCtuRe 1 (Beltrametti-Sommese). Let $(X, L)$ be an n-dimensional polarized manifold with $n \geq 3$. Assume that $K_{X}+(n-1) L$ is nef. Then $h^{0}\left(K_{X}+(n-1) L\right)>0$.

For this conjecture, the following partial results have been obtained.

(i) In [7, Theorem 2.4], the author proved that this conjecture is true if $n=3$.

(ii) In [13, 1.2 Theorem], Höring proved that this conjecture is true if $h^{0}(L)>0$.

2010 Mathematics Subject Classification. Primary 14C20; Secondary 14J35.

Key words and phrases. Polarized manifold, adjoint bundles, Beltrametti-Sommese conjecture, the sectional geometric genus.

This research was supported by JSPS KAKENHI Grant Number 24540043.

Received July 29, 2014; revised October 9, 2014. 
In this paper, we investigate the conjecture above, and the main purpose of this paper is to prove that the above conjecture is true for $n=4$.

We will use the customary notation in algebraic geometry.

\section{Preliminaries}

Notation 2.1. Let $X$ be a projective variety of dimension $n$ and let $L$ be a line bundle on $X$. Then we put

$$
\chi(t L)=\sum_{j=0}^{n} \chi_{j}(X, L)\left(\begin{array}{c}
t+j-1 \\
j
\end{array}\right)
$$

Definition 2.1 ([5, Definition 2.1]). Let $X$ be a projective variety of dimension $n$ and let $L$ be a line bundle on $X$.

(i) For every integer $i$ with $0 \leq i \leq n$, the $i$-th sectional geometric genus $g_{i}(X, L)$ of $(X, L)$ is defined by the following.

$$
g_{i}(X, L)=(-1)^{i}\left(\chi_{n-i}(X, L)-\chi\left(\mathcal{O}_{X}\right)\right)+\sum_{j=0}^{n-i}(-1)^{n-i-j} h^{n-j}\left(\mathcal{O}_{X}\right) .
$$

(ii) For every integer $i$ with $0 \leq i \leq n$, the $i$-th sectional $H$-arithmetic genus $\chi_{i}^{H}(X, L)$ of $(X, L)$ is defined by the following.

$$
\chi_{i}^{H}(X, L)=\chi_{n-i}(X, L) .
$$

Remark 2.1. (i) Since $\chi_{n-i}(X, L) \in \mathbf{Z}$, we see that $\chi_{i}^{H}(X, L)$ and $g_{i}(X, L)$ are integer by definition.

(ii) If $i=\operatorname{dim} X=n$, then $g_{n}(X, L)=h^{n}\left(\mathcal{O}_{X}\right)$ and $\chi_{n}^{H}(X, L)=\chi\left(\mathcal{O}_{X}\right)$.

(iii) If $i=0$, then $g_{0}(X, L)=L^{n}$ and $\chi_{0}^{H}(X, L)=L^{n}$.

(iv) If $i=1$, then $g_{1}(X, L)=g(X, L)$, where $g(X, L)$ is the sectional genus of $(X, L)$. If $X$ is smooth, then the sectional genus $g(X, L)$ can be written as

$$
g(X, L)=1+\frac{1}{2}\left(K_{X}+(n-1) L\right) L^{n-1} .
$$

(v) If $i=2$, then we have ${ }^{1}$

$$
\begin{aligned}
g_{2}(X, L)= & \frac{1}{12}\left(K_{X}+(n-1) L\right)\left(K_{X}+(n-2) L\right) L^{n-2}+\frac{1}{12} c_{2}(X) L^{n-2} \\
& +\frac{n-3}{24}\left(2 K_{X}+(n-2) L\right) L^{n-1}-1+h^{1}\left(\mathcal{O}_{X}\right) .
\end{aligned}
$$

\footnotetext{
${ }^{1}$ See $[6,(2.2 . \mathrm{A})]$.
} 
(vi) If $i=3$, then we have ${ }^{2}$

$$
\begin{aligned}
g_{3}(X, L)= & \frac{(n-2)(n-3)^{2}}{48} L^{n}+\frac{(n-3)(3 n-8)}{48} K_{X} L^{n-1} \\
& +\frac{n-3}{24}\left(K_{X}^{2}+c_{2}(X)\right) L^{n-2}+\frac{1}{24} K_{X} c_{2}(X) L^{n-3} \\
& +1-h^{1}\left(\mathcal{O}_{X}\right)+h^{2}\left(\mathcal{O}_{X}\right) .
\end{aligned}
$$

(vii) In general for every integer $i$ with $1 \leq i \leq n$ we get

$$
\chi_{i}^{H}(X, L)=1-h^{1}\left(\mathcal{O}_{X}\right)+\cdots+(-1)^{i-1} h^{i-1}\left(\mathcal{O}_{X}\right)+(-1)^{i} g_{i}(X, L) .
$$

THeOrem 2.1. Let $(X, L)$ be a polarized manifold with $\operatorname{dim} X=n$, and let $i$ be an integer with $0 \leq i \leq n-1$. Then

$$
g_{i}(X, L)=\sum_{j=0}^{n-i-1}(-1)^{j}\left(\begin{array}{c}
n-i \\
j
\end{array}\right) h^{0}\left(K_{X}+(n-i-j) L\right)+\sum_{k=0}^{n-i}(-1)^{n-i-k} h^{n-k}\left(\mathcal{O}_{X}\right) .
$$

Proof. See [5, Theorem 2.3].

Definition 2.2. (i) Let $X$ (resp. $Y$ ) be an $n$-dimensional projective manifold, and let $L$ (resp. $H$ ) be an ample line bundle on $X$ (resp. $Y$ ). Then $(X, L)$ is called a simple blowing up of $(Y, H)$ if there exists a birational morphism $\pi: X \rightarrow Y$ such that $\pi$ is a blowing up at a point of $Y$ and $L=\pi^{*}(H)-E$, where $E$ is the $\pi$-exceptional reduced divisor.

(ii) Let $X$ (resp. $M$ ) be an $n$-dimensional projective manifold, and let $L$ (resp. $A$ ) be an ample line bundle on $X$ (resp. $M)$. Then we say that $(M, A)$ is a reduction of $(X, L)$ if $(X, L)$ is obtained by a composite of simple blowing ups of $(M, A)$, and $(M, A)$ is not obtained by a simple blowing up of any polarized manifold. The morphism $\phi_{1}: X \rightarrow M$ is called the reduction map.

Remark 2.2. Let $(X, L)$ be a polarized manifold of dimension $n$ and $(M, A)$ a reduction of $(X, L)$.

(i) If $(X, L)$ is not obtained by a simple blowing up of another polarized manifold, then we regard $(X, L)$ as a reduction of itself.

(ii) For any polarized manifold $(X, L)$, there exists a reduction of $(X, L)$. Moreover if $n \geq 3$, then a reduction of $(X, L)$ is unique. (See [4, (11.11), Chapter II].)

(iii) If $\kappa\left(K_{X}+(n-2) L\right) \geq 0$, then we infer that $K_{M}+(n-2) A$ is nef (see [1, Proposition 7.2.2 and Theorems 7.2.3, 7.2.4, 7.3.2, 7.3.4]).

(iv) $h^{0}\left(K_{X}+t L\right)=h^{0}\left(K_{M}+t A\right)$ for every integer $t$ with $1 \leq t \leq n-1$.

\footnotetext{
${ }^{2}$ See $[6,(2.2 . \mathrm{B})]$
} 
Definition 2.3 ([9, Definition 3.1 and Definition 3.2]). Let $(X, L)$ be a polarized manifold of dimension $n$.

(i) Let $t$ be a positive integer. Then set

$$
\begin{aligned}
F_{0}(t) & :=h^{0}\left(K_{X}+t L\right), \\
F_{i}(t) & :=F_{i-1}(t+1)-F_{i-1}(t) \text { for every integer } i \text { with } 1 \leq i \leq n .
\end{aligned}
$$

(ii) For every integer $i$ with $0 \leq i \leq n$, the ith Hilbert coefficient $A_{i}(X, L)$ of $(X, L)$ is defined by $A_{i}(X, L)=F_{n-i}(1)$.

Remark 2.3. (i) If $1 \leq i \leq n$, then $A_{i}(X, L)$ can be written as follows (see [9, Proposition 3.2]).

$$
\begin{aligned}
A_{i}(X, L) & =(-1)^{i} \chi_{i}^{H}(X, L)+(-1)^{i-1} \chi_{i-1}^{H}(X, L) \\
& =g_{i}(X, L)+g_{i-1}(X, L)-h^{i-1}\left(\mathcal{O}_{X}\right) .
\end{aligned}
$$

(ii) By Definition 2.3 and [9, Proposition 3.1 (2)], we have the following: (ii.1) $A_{i}(X, L) \in \mathbf{Z}$ for every integer $i$ with $0 \leq i \leq n$.

(ii.2) $A_{0}(X, L)=L^{n}$.

(ii.3) $A_{n}(X, L)=h^{0}\left(K_{X}+L\right)$.

(iii) By Remark 2.1 (v) and (vi), and by Remark 2.3 (i), we see that $A_{2}(X, L)$ and $A_{3}(X, L)$ are the following.

$$
\begin{aligned}
A_{2}(X, L)= & \frac{(3 n-2)(n+1)}{24} L^{n}+\frac{n}{4} K_{X} L^{n-1}+\frac{1}{12}\left(K_{X}^{2}+c_{2}(X)\right) L^{n-2}, \\
A_{3}(X, L)= & \frac{(n-2)\left(n^{2}-1\right)}{48} L^{n}+\frac{n(3 n-5)}{48} K_{X} L^{n-1}+\frac{n-1}{24} K_{X}^{2} L^{n-2} \\
& +\frac{1}{24} c_{2}(X)\left(K_{X}+(n-1) L\right) L^{n-3} .
\end{aligned}
$$

THEOREM 2.2. Let $(X, L)$ be a polarized manifold of dimension $n$ and let $t$ be a positive integer. Then for every integer $i$ with $0 \leq i \leq n$ we have

$$
F_{n-i}(t)=\sum_{j=0}^{i}\left(\begin{array}{c}
t-1 \\
i-j
\end{array}\right) A_{j}(X, L) .
$$

Proof. See [9, Theorem 3.1].

Corollary 2.1 ([9, Corollary 3.1]). Let $(X, L)$ be a polarized manifold of dimension $n$, and let $t$ be a positive integer. Then we have

$$
h^{0}\left(K_{X}+t L\right)=\sum_{j=0}^{n}\left(\begin{array}{c}
t-1 \\
n-j
\end{array}\right) A_{j}(X, L) .
$$


Remark 2.4. Let $(X, L)$ be a polarized manifold of dimension $n$. Then by using $A_{i}(X, L)$, the left hand side of equations (6) and (7) in [13, 4.1 Lemma] can be written as follows.

$$
\begin{gathered}
\chi\left(\mathcal{O}_{X}\right)+\frac{1}{2}\left(K_{X}+(n-1) L\right) L^{n-1}=\sum_{i=2}^{n}(-1)^{i} A_{i}(X, L), \\
L^{n-2}\left(2\left(K_{X}^{2}+c_{2}(X)\right)+6 n K_{X} L+(n+1)(3 n-2) L^{2}\right)=24 A_{2}(X, L) .
\end{gathered}
$$

THEOREM 2.3. Let $X$ be a projective manifold. Then there exist smooth projective varieties $X^{\prime}$ and $Y$, a birational morphism $\mu: X^{\prime} \rightarrow X$ and a fiber space $\phi: X^{\prime} \rightarrow Y$ such that $Y$ is not uniruled and if $\operatorname{dim} X^{\prime}>\operatorname{dim} Y$, then the general fiber of $\phi$ is rationally connected.

Proof. See [2], [14] and [11].

Definition 2.4. The fiber space $\phi: X^{\prime} \rightarrow Y$ in Theorem 2.3 is called the $M R C$-fibration of $X$, and $Y$ is called the base of the MRC-fibration.

\section{Main result}

In this section we are going to prove Conjecture 1 for the case of dimension 4.

THEOREM 3.1. Let $(X, L)$ be a polarized manifold of dimension 4. Assume that $K_{X}+3 L$ is nef. Then $h^{0}\left(K_{X}+3 L\right)>0$ holds.

Proof. (I) First we consider the case where $q(X)>0$ (see also the proof of $[8$, Theorem 3.3]). Let $\alpha: X \rightarrow \operatorname{Alb}(X)$ be the Albanese map. Then $1 \leq \operatorname{dim} \alpha(X) \leq 4$. Then by [12, Corollary 10.7, Chapter III, Section 10], a general fiber $F_{\alpha}$ of $\alpha$ is the following type:

$$
F_{\alpha}=\bigcup_{j=1}^{r} F_{j},
$$

where $F_{j}$ is a smooth subvariety for every integer $j$ with $1 \leq j \leq r, \operatorname{dim} F_{k}=$ $\operatorname{dim} F_{l}$ and $F_{k} \cap F_{l}=\emptyset$ for any $k \neq l$. Here we note that if $\kappa\left(K_{X}+m L\right) \geq 0$, then $\kappa\left(K_{F_{j}}+m L_{F_{j}}\right) \geq 0$ for every integer $j$ with $1 \leq j \leq r$. We also note that $0 \leq \operatorname{dim} F_{j} \leq 3$ for every $j$.

(I.1) If $\operatorname{dim} F_{\alpha}=0$, then $h^{0}\left(K_{F_{j}}+3 L_{F_{j}}\right)>0$ for every integer $j$ with $1 \leq$ $j \leq r$.

(I.2) Assume that $\operatorname{dim} F_{\alpha}=3$ (resp. $1 \leq \operatorname{dim} F_{\alpha} \leq 2$ ). Since $K_{F_{j}}+3 L_{F_{j}}$ is nef by assumption, we see from [13, 1.5 Theorem] (resp. [8, Theorem 2.8]) that $h^{0}\left(K_{F_{j}}+3 L_{F_{j}}\right)>0$. Hence $h^{0}\left(K_{F_{\alpha}}+3 L_{F_{\alpha}}\right)>0$. So by [3, Lemma 4.1], we have $h^{0}\left(K_{X}+3 L\right)>0$ and we get the assertion. 
(II) Next we consider the case where $q(X)=0$.

(II.1) If $\kappa\left(K_{X}+2 L\right)=-\infty$, then $h^{0}\left(K_{X}+t L\right)=0$ for $t=1,2$. So we get the assertion by $[13,1.2$ Theorem].

(II.2) Assume that $\kappa\left(K_{X}+2 L\right) \geq 0$. By taking the reduction of $(X, L)$, if necessary, we may assume that $K_{X}+2 L$ is nef by Remark 2.2 (iii) and (iv).

(II.2.1) Assume that $\Omega_{X}\langle L\rangle$ is generically nef. Since $K_{X}+4 L$ is ample by assumption, we see from $[13,2.11$ Corollary $]$ that

$$
\begin{aligned}
c_{2}(X)\left(K_{X}+4 L\right) L & \geq-\left(\frac{3}{4} K_{X}(4 L)+\frac{3}{8}(4 L)^{2}\right)\left(K_{X}+4 L\right) L \\
& =-3 K_{X}^{2} L^{2}-18 K_{X} L^{3}-24 L^{4} .
\end{aligned}
$$

Here we calculate $A_{2}(X, L)+2 A_{3}(X, L)$. By Remark 2.3 (iii) we have

$$
\begin{aligned}
A_{2}(X, L) & +2 A_{3}(X, L) \\
= & \frac{1}{12}\left(K_{X}+3 L\right)\left(K_{X}+8 L\right) L^{2}+\frac{1}{24}\left(2 K_{X}+2 L\right) L^{3} \\
& +\frac{5}{4} L^{4}+\frac{7}{6} K_{X} L^{3}+\frac{1}{4} K_{X}^{2} L^{2}+\frac{1}{12} c_{2}(X)\left(K_{X}+4 L\right) L \\
= & \frac{1}{3} K_{X}^{2} L^{2}+\frac{13}{6} K_{X} L^{3}+\frac{10}{3} L^{4}+\frac{1}{12} c_{2}(X)\left(K_{X}+4 L\right) L .
\end{aligned}
$$

Hence by (3) and (4) we have

$$
\begin{aligned}
A_{2}(X, L)+2 A_{3}(X, L) \\
=\frac{1}{3} K_{X}^{2} L^{2}+\frac{13}{6} K_{X} L^{3}+\frac{10}{3} L^{4}+\frac{1}{12} c_{2}(X)\left(K_{X}+4 L\right) L \\
\geq \frac{1}{3} K_{X}^{2} L^{2}+\frac{13}{6} K_{X} L^{3}+\frac{10}{3} L^{4}-\frac{1}{12}\left(3 K_{X}^{2} L^{2}+18 K_{X} L^{3}+24 L^{4}\right) \\
=\frac{1}{12} K_{X}^{2} L^{2}+\frac{8}{12} K_{X} L^{3}+\frac{4}{3} L^{4} \\
=\frac{1}{12}\left(K_{X}+2 L\right)^{2} L^{2}+\frac{1}{3}\left(K_{X}+3 L\right) L^{3} .
\end{aligned}
$$

Since $K_{X}+2 L$ is nef by assumption, we have $A_{2}(X, L)+2 A_{3}(X, L)>0$. Here we note that $A_{4}(X, L)=h^{0}\left(K_{X}+L\right) \geq 0$ by Remark 2.3 (ii.3). Therefore

$$
h^{0}\left(K_{X}+3 L\right)=A_{4}(X, L)+2 A_{3}(X, L)+A_{2}(X, L)>0 .
$$

(II.2.2) Assume that $\Omega_{X}\langle L\rangle$ is not generically nef. Then by [13, 3.1 Theorem] there exist smooth projective varieties $X^{\prime}$ and $Y$, a birational morphism $\mu: X^{\prime} \rightarrow X$ and a fiber space $\lambda: X^{\prime} \rightarrow Y$ such that $m:=\operatorname{dim} Y<4$ and a general fiber $F_{\lambda}$ of $\lambda$ is rationally connected and $h^{0}(D)=0$ for any Cartier divisor $D$ on $F_{\lambda}$ with $D \sim_{\mathbf{Q}} K_{F_{\lambda}}+j\left(\mu^{*}(L)\right)_{F_{\lambda}}$ with $j \in[0,4-m] \cap \mathbf{Q}$. 
(II.2.2.1) The case where $\operatorname{dim} Y=0$ or 1 . Then $h^{0}\left(K_{X}+t L\right)=h^{0}\left(K_{X^{\prime}}+\right.$ $\left.\mu^{*}(t L)\right)=0$ for $t=1,2,3$. But this is impossible by [13, 1.2 Theorem].

(II.2.2.2) The case where $\operatorname{dim} Y=2$. Then we have $h^{0}\left(K_{F_{\lambda}}+\mu^{*}(2 L)_{F_{\lambda}}\right)=0$. On the other hand, since $\kappa\left(K_{X}+2 L\right) \geq 0$, we have $\kappa\left(K_{F_{\lambda}}+\mu^{*}(2 L)_{F_{2}}\right) \geq 0$. Here we note that $\operatorname{dim} F_{\lambda}=2$. Hence $h^{0}\left(K_{F_{\lambda}}+\mu^{*}(2 L)_{F_{\lambda}}\right)>0$ by [10, Proposition 1] (see also [8, Theorem 2.8]). But this is a contradiction.

(II.2.2.3) The case where $\operatorname{dim} Y=3$. In this case $F_{\lambda} \cong \mathbf{P}^{1}$. Since $h^{0}(D)=0$ for any Cartier divisor $D$ on $F_{\lambda}$ with $D \sim_{\mathbf{Q}} K_{F_{\lambda}}+\mu^{*}(L)_{F_{2}}$, we have $\operatorname{deg} \mu^{*}(L)_{F_{\lambda}}=1$. In this case $h^{0}\left(K_{X}+L\right)=0$ and $h^{4}\left(\mathcal{O}_{X}\right)=0$ hold. Hence by Theorem 2.1 we obtain

$$
\begin{aligned}
& g_{4}(X, L)=0, \\
& g_{3}(X, L)=h^{3}\left(\mathcal{O}_{X}\right), \\
& g_{2}(X, L)=h^{0}\left(K_{X}+2 L\right)+h^{2}\left(\mathcal{O}_{X}\right)-h^{3}\left(\mathcal{O}_{X}\right) .
\end{aligned}
$$

Hence by (6), (7) and Remark 2.3 (i) we have $A_{3}(X, L)=h^{0}\left(K_{X}+2 L\right)$. By assumption we have

$$
h^{1}\left(\mathcal{O}_{X}\right)=0 .
$$

Since $h^{0}\left(K_{X}+L\right)=0$, we see from [13, 1.2 Theorem] that we get $h^{0}\left(K_{X}+2 L\right)$ $>0$ or $h^{0}\left(K_{X}+3 L\right)>0$. If $h^{0}\left(K_{X}+3 L\right)>0$, then we get the assertion. So we may assume that

$$
h^{0}\left(K_{X}+2 L\right)>0 \quad \text { and } \quad h^{0}\left(K_{X}+3 L\right)=0 .
$$

We note that by Definition 2.3 (i) we get $F_{0}(t)=h^{0}\left(K_{X}+t L\right), \quad F_{1}(t)=$ $F_{0}(t+1)-F_{0}(t)=h^{0}\left(K_{X}+(t+1) L\right)-h^{0}\left(K_{X}+t L\right)$. Hence

$$
\begin{aligned}
F_{1}(2) & =F_{0}(3)-F_{0}(2) \\
& =h^{0}\left(K_{X}+3 L\right)-h^{0}\left(K_{X}+2 L\right) \\
& <0 .
\end{aligned}
$$

On the other hand, by Theorem 2.2 we get

$$
F_{4-i}(t)=\sum_{j=0}^{i}\left(\begin{array}{l}
t-1 \\
i-j
\end{array}\right) A_{j}(X, L) .
$$

Therefore

$$
0>F_{1}(2)=A_{2}(X, L)+A_{3}(X, L)
$$

Furthermore

$$
F_{1}(1)=h^{0}\left(K_{X}+2 L\right)-h^{0}\left(K_{X}+L\right)>0
$$

and by Theorem 2.2 we have

$$
F_{1}(1)=A_{3}(X, L) .
$$


So we get

$$
A_{3}(X, L)>0
$$

By (10) and (11) we have

$$
A_{2}(X, L)<0 .
$$

Here we prove the following claim. least 3 .

Claim 3.1. The dimension of the base of the $M R C$-fibration ${ }^{3}$ of $X$ is at

Proof. Assume that the dimension of the base of the MRC-fibration is less than or equal to two. Then we have

$$
h^{j}\left(\mathcal{O}_{X}\right)=0 \text { for every integer } j \geq 3 \text {. }
$$

First we note that since $g_{4}(X, L)=0$ and $g_{3}(X, L)=h^{3}\left(\mathcal{O}_{X}\right)$ by (5) and (6), we see from Remark 2.3 (i) that

$$
A_{4}(X, L)=g_{4}(X, L)+g_{3}(X, L)-h^{3}\left(\mathcal{O}_{X}\right)=0 .
$$

So by (11), (12) and (14) we have

$$
\sum_{i=2}^{4}(-1)^{i} A_{i}(X, L)<0 .
$$

On the other hand by Remark 2.4 (1) we have

$$
\sum_{i=2}^{4}(-1)^{i} A_{i}(X, L)=\chi\left(\mathcal{O}_{X}\right)+\frac{1}{2}\left(K_{X}+3 L\right) L^{3} .
$$

Since $\left(K_{X}+3 L\right) L^{3}>0$ in this case, we have $\chi\left(\mathcal{O}_{X}\right)<0$. So we see from (13) that $h^{1}\left(\mathcal{O}_{X}\right)>0$ and this contradicts the assumption (8). So we get the assertion of Claim 3.1.

By Claim 3.1 and the argument of [13, Step 2 in Page 741], we see from Remark 2.4 (2) that $^{4}$

$$
A_{2}(X, L)=\frac{1}{24} L^{2}\left(2\left(K_{X}^{2}+c_{2}(X)\right)+24 K_{X} L+50 L^{2}\right)>0
$$

which contradicts (12). Therefore the assumption (9) is impossible.

Therefore we get the assertion of Theorem 3.1.

\footnotetext{
${ }^{3}$ See Definition 2.4 .

${ }^{4}$ Here we note that $n=4$ in this case.
} 
Acknowledgment. The author would like to thank the referee for giving valuable suggestions.

\title{
REFERENCES
}

[1] M. C. Beltrametti and A. J. Sommese, The adjunction theory of complex projective varieties, de Gruyter expositions in math. 16, Walter de Gruyter, Berlin, New York, 1995.

[2] F. Campana, Connexite rationnelle des varietes de Fano, Ann. Sci. Ecole Norm. Sup. (4) 25 (1992), 539-545.

[3] J. A. Chen and C. D. Hacon, Linear series of irregular varieties, Algebraic geometry in East Asia, Kyoto 2001, World Sci. Publishing, River Edge, NJ, 2002, 143-153.

[4] T. Fustra, Classification theories of polarized varieties, London Math. Soc. lecture note series 155, Cambridge University Press, Cambridge, 1990.

[5] Y. Fukuma, On the sectional geometric genus of quasi-polarized varieties, I, Comm. Alg. 32 (2004), 1069-1100.

[6] Y. Fukuma, A formula for the sectional geometric genus of quasi-polarized manifolds by using intersection numbers, J. Pure Appl. Algebra 194 (2004), 113-126.

[ 7 ] Y. Fukuma, On a conjecture of Beltrametti-Sommese for polarized 3-folds, Internat. J. Math. 17 (2006), 761-789.

[ 8 ] Y. Fukuma, On the dimension of global sections of adjoint bundles for polarized 3-folds and 4-folds, J. Pure Appl. Algebra 211 (2007), 609-621.

[9] Y. Fukuma, A study on the dimension of global sections of adjoint bundles for polarized manifolds, J. Algebra 320 (2008), 3543-3558.

[10] Y. Fukuma, A lower bound for the second sectional geometric genus of quasi-polarized manifolds and its applications, Rend. Semin. Mat. Univ. Politec. Torino 69 (2011), 73-90.

[11] T. Graber, J. Harris and J. Starr, Families of rationally connected varieties, J. Amer. Math. Soc. 16 (2003), 57-67.

[12] R. Hartshorne, Algebraic geometry, Graduate texts in math. 52, Springer-Verlag, New York, 1977.

[13] A. Höring, On a conjecture of Beltrametti and Sommese, J. Algebraic Geom. 21 (2012), $721-751$.

[14] J. Kollár, Y. Miyaoka and S. Mori, Rational connectedness and boundedness of Fano manifolds, J. Differential Geom. 36 (1992), 765-779.

\author{
Yoshiaki Fukuma \\ DePARTMENT OF Mathematics \\ FACULTY OF SCIENCE \\ KOCHI UNIVERSITY \\ AKEBONO-CHO, KOCHI 780-8520 \\ JAPAN \\ E-mail: fukuma@kochi-u.ac.jp
}

